\title{
Spontaneous rhythms in a harbor seal pup calls
}

\author{
Andrea Ravignani $i^{1,2,3^{*}}$
}

\begin{abstract}
Objectives: Timing and rhythm (i.e. temporal structure) are crucial, though historically neglected, dimensions of animal communication. When investigating these in non-human animals, it is often difficult to balance experimental control and ecological validity. Here I present the first step of an attempt to balance the two, focusing on the timing of vocal rhythms in a harbor seal pup (Phoca vitulina). Collection of this data had a clear aim: To find spontaneous vocal rhythms in this individual in order to design individually-adapted and ecologically-relevant stimuli for a later playback experiment.

Data description: The calls of one seal pup were recorded. The audio recordings were annotated using Praat, a free software to analyze vocalizations in humans and other animals. The annotated onsets and offsets of vocalizations were then imported in a Python script. The script extracted three types of timing information: the duration of calls, the intervals between calls' onsets, and the intervals between calls' maximum-intensity peaks. Based on the annotated data, available to download, I provide simple descriptive statistics for these temporal measures, and compare their distributions.
\end{abstract}

Keywords: Bioacoustics, Rhythm, Vocal communication, Vocalization, Animal call, Marine mammal, Pinniped, Turntaking, Timing, Evolution of speech, Harbor seal

\section{Objective}

Pinnipeds are a clade of marine mammals exhibiting a range of vocal behaviors $[1,2]$. Testing rhythm and timing in pinnipeds is relevant to a number of cross-species evolutionary hypotheses relating rhythmic behaviors to vocal flexibility, social cognition, and brain plasticity [3-8].

Traditionally, the comparative study of rhythm and timing has spanned two main strands. Animal vocalizations and behaviors have been recorded in ecologicallyrelevant settings to unveil temporal structures. These observational approaches allowed little experimental control. Alternatively, animals have been tested in an operant setup, employing controlled external stimuli to trigger animals' reactions. These other approaches traded ecological relevance for experimental control. While

${ }^{*}$ Correspondence: andrea.ravignani@gmail.com

${ }^{1}$ Research Department, Sealcentre Pieterburen, Hoofdstraat 94a, 9968

AG Pieterburen, The Netherlands

Full list of author information is available at the end of the article a few exceptions exist [9], animal research on rhythm and timing still needs to strike a good tradeoff between experimental rigor and ecological relevance.

Here I present the first step of an attempt to balance the two. I describe and share data on spontaneous vocal rhythms in a harbor seal pup. Audio recordings were collected with the explicit aim of finding the natural timing of vocal production in this individual, and design ecologically-relevant and individually-adapted stimuli for a future playback experiment [9-11].

After recording the animal, I annotated the onsets and offsets of vocalizations in Praat. Using a custom Python script (Table 1, Data file 1) [12,13], I extracted three sorts of timing information: the duration of calls [14-17], the intervals between calls' onsets $[18,19]$, and the intervals between calls' maximum-intensity peaks [20].

\section{Data description}

\section{Subject}

I recorded a female harbor seal pup. The seal was born in the wild and brought into rehabilitation at the Sealcentre 
Table 1 Overview of data files/data sets

\begin{tabular}{|c|c|c|c|c|}
\hline Label & Name of data file/data set & File types (file extension) & Data repository and identifier (DOI or accession number) & License \\
\hline Data file 1 & textgrid_IOI_IPI_dur_01 & py & Figshare (https://doi.org/10.6084/m9.figshare.5616490) & $C C-B Y$ \\
\hline Data file 2 & durations_all &. $\operatorname{csv}$ & Figshare (https://doi.org/10.6084/m9.figshare.5616490) & $C C-B Y$ \\
\hline Data file 3 & IOI_all &. $\operatorname{csv}$ & Figshare (https://doi.org/10.6084/m9.figshare.5616490) & $C C-B Y$ \\
\hline Data file 4 & IPI_all &. $\operatorname{csv}$ & Figshare (https://doi.org/10.6084/m9.figshare.5616490) & $C C-B Y$ \\
\hline Data file 5 & 1OI_short &. $\operatorname{csV}$ & Figshare (https://doi.org/10.6084/m9.figshare.5616490) & $C C-B Y$ \\
\hline Data file 6 & IPI_short & . CSV & Figshare (https://doi.org/10.6084/m9.figshare.5616490) & $C C-B Y$ \\
\hline Data file 7 & supplement_datanote01 & docx & Figshare (https://doi.org/10.6084/m9.figshare.5616490) & $C C-B Y$ \\
\hline
\end{tabular}

Pieterburen, The Netherlands [21, 22], at the estimated age of 7 days $[14,22]$. The animal was individually housed in a pool situated in a 1-room cabin. Seals in rehabilitation are usually housed in pairs [14]; this recording exploited the rare occurrence of individual housing.

\section{Sound recordings}

On the twenty-first day from estimated birth, $10 \mathrm{~min}$ of vocalizations were recorded in air using a unidirectional microphone Sennheiser ME-66 (frequency response: 40-20,000 Hz $\pm 2.5 \mathrm{~dB}$; Sennheiser electronic GmbH\&Co. KG, Wedemark, Germany) [14]. The microphone was equipped with a MZW-66 foam windshield, and was connected to a digital recorder Zoom H6 (Zoom Corporation, Tokyo, Japan). Recordings, collected at $0.5-2 \mathrm{~m}$ distance from the seal, were saved as a .wav file (48 kHz sampling frequency; 24-bit quantization).

\section{Call annotations}

The audio file was manually annotated in Praat version 6.0.11 [23]. Mother attraction calls (MACs) and other calls were annotated as two different categories on one tier. The tier was saved as a .TextGrid file. Only clear MACs $[10,11,17,24]$ were retained for further computations $[14,15,17]$.

\section{Extraction of temporal variables}

A Python 2.7 script extracted and combined annotations and sound features (Table 1, Data file 1), and outputted five .csv files. The script imported the annotations using package TextGridTools 1.4.3 [12] and the wave sound using Parselmouth [13]. The script calculated: durations (Table 1, Data file 2), inter-onset intervals (IOIs), and inter-peak intervals (IPIs) of calls. An IOI was defined as the time elapsed between the onsets of two consecutive calls (Table 1, Data file 3). An IPI was defined as the time between the maximum-intensity peaks of two consecutive calls (Table 1, Data file 4) [20]. Two more datasets were computed and output: short IOIs (IOI ${ }^{\mathrm{s}}$, Table 1, Data file 5) and short IPIs (IPI ${ }^{\mathrm{s}}$, Table 1, Data file 6), consisting of intervals within approximately 4 times the minimum value $(\approx 3900 \mathrm{~ms}$ ). The purpose of this threshold was to focus on timing within vocalization bouts $\left(\mathrm{IOI}^{\mathrm{s}}\right.$ and $\left.\mathrm{IPI}^{\mathrm{s}}\right)$ as opposed to pooled timing within and between bouts (IOI and IPI).

\section{Descriptive statistics}

Mean call duration was $976.1 \mathrm{~ms}$ (standard deviation $\boldsymbol{\sigma}=205.7$, see also Table 1, Data file 7). Mean IOI was $8578.3 \mathrm{~ms}(\sigma=7807.4)$. Mean IPI was $8574.6 \mathrm{~ms}$ $(\sigma=7839.8)$. No significant difference was detected between these two distributions (Two-sample Kolmogorov-Smirnov test, $\mathrm{D}=0.04, \mathrm{p}=0.99)$. Mean $\mathrm{IOI}^{\mathrm{s}}$ was $1983.2 \mathrm{~ms}(\sigma=722.1)$. Mean $\mathrm{IPI}^{\mathrm{s}}$ was $2020.8 \mathrm{~ms}$ $(\sigma=803.3)$. No significant difference was detected between distributions of $\mathrm{IOI}^{\mathrm{s}} \mathrm{s}$ and $\mathrm{IPI}^{\mathrm{s}} \mathrm{s}$ (Two-sample Kolmogorov-Smirnov test, $\mathrm{D}=0.10, \mathrm{p}=0.99$ ). In other words, using onsets instead of peaks does not yield a significant difference between distributions. This holds at two different timescales, i.e. for both the IOI/IPI and the $\mathrm{IOI}^{\mathrm{s}} / \mathrm{IPI}^{\mathrm{s}}$ comparisons. The distributions of IOI and IPI have very high $\sigma$, almost equal to their means ( $\mathrm{CV}$, coefficient of variation, equals 0.91 for IOI and IPI). Conversely, the distributions of $\mathrm{IOI}^{\mathrm{s}}$ and $\mathrm{IPI}^{\mathrm{s}}$ have lower $\boldsymbol{\sigma}$ (CV equals 0.36 for $\mathrm{IOI}^{\mathrm{s}}$ and 0.39 for $\mathrm{IPI}^{\mathrm{S}}$ ).

\section{Limitations}

A clear limitation of these data is their focus on one individual. Pups in rehabilitation are usually kept in groups. Hence, it is uncommon to record long runs of vocalizations from isolated individuals. Data from this individual seal served its purpose of tailoring an experiment to her [25]. However, solid inference about rhythm ontogeny, learning, individual differences, and species differences will require additional data [7]. It would be desirable to collect a panel dataset, where multiple animals are recorded daily, showing variance over individuals and time. Such dataset would enable comparing the (1) type of temporal distributions, (2) average length, and (3) degree of isochronous regularity, both between species 
(e.g. Phocids vs. Otariids $[6,7,18]$ ) and between housing conditions (lonely vs. in-pair housing). Seal pups' call duration is known to vary with age [14, 15, 17]; all the other temporal variables presented in this Data Note are rarely investigated in pinniped bioacoustics.

A second limitation is that vocalizations were exclusively recorded in-air, while harbor seal pups [17] and adults [16] also vocalize underwater. This might not be an issue, because the medium of sound transmission should affect spectral, rather than temporal, properties of the calls [6]. However, vocal production repertoires might also vary across media, with some vocalizations only appearing in-air or underwater. Past research found that call duration is comparable across media [17]; comparison of IOIs and IPIs across media remains, to my knowledge, unexplored.

To comply with Data Note articles' guidelines, this paper lacks analyses. Although simple descriptive statistics are suited for the original purpose of these recordings, namely estimating the mean $\mathrm{IOI}^{\mathrm{s}}$ and using it to produce experimental stimuli, some analyses could be performed $[19,26]$.

\section{Abbreviations \\ MAC: mother attraction calls; IOI: inter-onset interval; IOI's short inter-onset interval; IPI: inter-peak interval; ||$^{\text {s. }}$ : short inter-peak interval; $\sigma$ : one standard deviation from the mean; $\mathrm{CV}$ : coefficient of variation.}

\section{Author details}

1 Research Department, Sealcentre Pieterburen, Hoofdstraat 94a, 9968 AG Pieterburen, The Netherlands. ${ }^{2}$ Artificial Intelligence Lab, Vrije Universiteit Brussel, Pleinlaan 2, 1050 Brussels, Belgium. ${ }^{3}$ Language and Cognition Department, Max Planck Institute for Psycholinguistics, Wundtlaan 1, 6525 XD Nijmegen, The Netherlands.

\section{Acknowledgements}

The author is grateful to Peter Cook, Bart de Boer, Koen de Reus, Sonja Kotz, Marga Mendez-Arostegui, Ana Rubio-Garcia, Marianna Anichini, and Ruth Sonnweber for advice on the research and/or manuscript.

\section{Competing interests}

The author declares that he has no competing interests.

\section{Availability of data materials}

The data described in this Data note can be freely and openly accessed on Figshare https://doi.org/10.6084/m9.figshare.5616490. Please see Table 1 and reference list for details and links to the data.

\section{Consent for publication}

Not applicable.

\section{Ethics approval and consent to participate}

The audio recordings were non-invasive and did not require ethical approval. Data collection was in accordance with Belgian, Dutch, and European Union legislation, and the guidelines of the Association for the Study of Animal Behavior.

\section{Funding}

AR has received funding from the European Union's Horizon 2020 research and innovation programme under the Marie Skłodowska-Curie Grant Agreement No. 665501 with the research Foundation Flanders (FWO) (Pegasus²
Marie Curie fellowship 12N5517N), and a visiting fellowship in Language Evolution from the Max Planck Society.

\section{Publisher's Note}

Springer Nature remains neutral with regard to jurisdictional claims in published maps and institutional affiliations.

Received: 21 November 2017 Accepted: 19 December 2017

Published online: 03 January 2018

\section{References}

1. Reichmuth C, Casey C. Vocal learning in seals, sea lions, and walruses. Curr Opin Neurobiol. 2014;28:66-71.

2. Schusterman R, Southall B, Kastak D, Reichmuth Kastak C. Pinniped vocal communication: Form and function. In: Proceedings of the 17th International Congress on Acoustics, Rome, Italy; 2001. p. 1-2.

3. Cook P, Rouse A, Wilson M, Reichmuth CJ. A California Sea Lion (Zalophus californianus) can keep the beat: motor entrainment to rhythmic auditory stimuli in a non vocal mimic. J Comp Psychol. 2013;127(2):1-16.

4. Patel AD. Musical rhythm, linguistic rhythm, and human evolution. Music Percept Interdiscip J. 2006;24(1):99-104.

5. Patel AD. The evolutionary biology of musical rhythm: was Darwin wrong? PLoS Biol. 2014;12(3):e1001821.

6. Ravignani A. Comment on "Temporal and spatial variation in harbor seal (Phoca vitulina L.) roar calls from southern Scandinavia" [J. Acoust. Soc Am. 141, 1824-1834 (2017)]. J Acoust Soc Am. (in review).

7. Ravignani A, Fitch WT, Hanke FD, Heinrich T, Hurgitsch B, Kotz SA, Scharff C, Stoeger AS, de Boer B. What pinnipeds have to say about human speech, music, and the evolution of rhythm. Front Neurosci. 2016;10(274):1-9.

8. Ralls K, Fiorelli P, Gish S. Vocalizations and vocal mimicry in captive harbor seals, Phoca vitulina. Can J Zool. 1985;63(5):1050-6.

9. Mathevon N, Casey C, Reichmuth C, Charrier I. Northern elephant seals memorize the rhythm and timbre of their rivals'voices. Curr Biol. 2017;27(15):2352-6.

10. Perry EA, Renouf D. Further studies of the role of harbour seal (Phoca vitulina) pup vocalizations in preventing separation of mother-pup pairs. Can J Zool. 1988;66(4):934-8.

11. Sauvé CC, Beauplet G, Hammill MO, Charrier I. Mother-pup vocal recognition in harbour seals: influence of maternal behaviour, pup voice and habitat sound properties. Anim Behav. 2015;105:109-20.

12. Buschmeier H, Wlodarczak M. TextGridTools: A TextGrid processing and analysis toolkit for Python. In: Proceedings der 27 Konferenz zur Elektronischen Sprachsignalverarbeitung. Bielefeld; 2013. p. 152-7.

13. Jadoul Y, Thompson B, de Boer B. Introducing Parselmouth: a Python interface to Praat. J Phon (in review).

14. de Reus K. Talking seals: vocal development in Eastern Atlantic harbour seal pups (Phoca vitulina vitulina). M.Sc. Royal Veterinary College, University of London; 2017.

15. Khan CB, Markowitz H, McCowan B. Vocal development in captive harbor seal pups, Phoca vitulina richardii: age, sex, and individual differences. J Acoust Soc Am. 2006;120(3):1684-94.

16. Sabinsky PF, Larsen ON, Wahlberg M, Tougaard J. Temporal and spatial variation in harbor seal (Phoca vitulina L.) roar calls from southern Scandinavia. J Acoust Soc Am. 2017;141 (3):1824-34.

17. Sauvé CC, Beauplet G, Hammill MO, Charrier I. Acoustic analysis of airborne, underwater, and amphibious mother attraction calls by wild harbor seal pups (Phoca vitulina). J Mammal. 2015;96(3):591-602.

18. Ravignani A, Madison $G$. The paradox of isochrony in the evolution of human rhythm. Front Psychol. 1820;2017(8):1-13.

19. Ravignani A, Norton P. Measuring rhythmic complexity: a primer to quantify and compare temporal structure in speech, movement, and animal vocalizations. J Lang Evol. 2017;2(1):4-19.

20. Jadoul Y, Ravignani A, Thompson B, Filippi P, de Boer B. Seeking temporal predictability in speech: comparing statistical approaches on 18 world languages. Front Hum Neurosci. 2016;10(586):1-15. 
21. Osinga N, Pen I, de Haes HU, Brakefield P. Evidence for a progressively earlier pupping season of the common seal (Phoca vitulina) in the Wadden Sea. J Mar Biol Assoc UK. 2012;92(08):1663-8.

22. Ravignani A, Gross S, Garcia M, Rubio-Garcia A, de Boer B. How small could a pup sound? The physical bases of signaling body size in harbor seals. Curr Zool. 2017;63(4):457-65.

23. Boersma, Weenink. 2017. http://www.praat.org/. Accessed 1 Jan 2017.

24. Renouf D. The vocalization of the harbour seal pup (Phoca vitulina) and its role in the maintenance of contact with the mother. J Zool. 1984;202(4):583-90
25. Ravignani A. A selfish seal chorus: timing of contagious calling in a harbor seal pup (in preparation).

26. Kello CT, Dalla Bella S, Médé B, Balasubramaniam R. Hierarchical temporal structure in music, speech and animal vocalizations: jazz is like a conversation, humpbacks sing like hermit thrushes. J R Soc Interface. 2017;14(135):20170231

\section{Submit your next manuscript to BioMed Central and we will help you at every step:}

- We accept pre-submission inquiries

- Our selector tool helps you to find the most relevant journal

- We provide round the clock customer support

- Convenient online submission

- Thorough peer review

- Inclusion in PubMed and all major indexing services

- Maximum visibility for your research

Submit your manuscript at

www.biomedcentral com/submit 